\title{
Transcriptome signature of the adult mouse choroid plexus
}

\author{
Fernanda Marques', João C Sousa ${ }^{1}$, Giovanni Coppola², Fuying Gao², Renato Puga ${ }^{3}$, Helena Brentani ${ }^{3}$, \\ Daniel H Geschwind ${ }^{2}$, Nuno Sousa ${ }^{1}$, Margarida Correia-Neves ${ }^{1}$, Joana A Palha ${ }^{1 *}$
}

\begin{abstract}
Background: Although the gene expression profile of several tissues in humans and in rodent animal models has been explored, analysis of the complete choroid plexus (CP) transcriptome is still lacking. A better characterization of the CP transcriptome can provide key insights into its functions as one of the barriers that separate the brain from the periphery and in the production of cerebrospinal fluid.

Methods: This work extends further what is known about the mouse CP transcriptome through a microarray analysis of CP tissue from normal mice under physiological conditions.

Results: We found that the genes most highly expressed are those implicated in energy metabolism (oxidative phosphorylation, glycolysis/gluconeogenesis) and in ribosomal function, which is in agreement with the secretory nature of the CP. On the other hand, genes encoding for immune mediators are among those with lower expression in basal conditions. In addition, we found genes known to be relevant during brain development, and not previously identified to be expressed in the $\mathrm{CP}$, including those encoding for various axonal guidance and angiogenesis molecules and for growth factors. Some of these are known to influence the neural stem cell niche in the subventricular zone, highlighting the involvement of the CP as a likely modulator of neurogenesis. Interestingly, our observations confirm that the CP transcriptome is unique, displaying low homology with that of other tissues. Of note, we describe here that the closest similarity is with the transcriptome of the endothelial cells of the blood-brain barrier.
\end{abstract}

Conclusions: Based on the data presented here, it will now be possible to further explore the function of particular proteins of the CP secretome in health and in disease.

\section{Background}

The choroid plexus $(\mathrm{CP})$ is located in the ventricles of the vertebrate brain. It is formed by a monolayer of epithelial cells that surrounds a central stroma in which fenestrated blood vessels are embedded in a rich extracellular matrix. In addition, and depending on the physiological or pathological conditions, the stroma also contains cells such as fibroblasts, macrophages, neutrophils, dendritic cells, plus B and T cells. Tight junctions between the apical sides of the epithelial cells separate the blood from the cerebrospinal fluid (CSF); this provides the barrier function that normally hinders the free movement of molecules and cells from the blood into

\footnotetext{
* Correspondence: japalha@ecsaude.uminho.pt

'Life and Health Sciences Research Institute (ICVS), School of Health

Sciences, University of Minho, Campus Gualtar, 4710-057 Braga, Portugal

Full list of author information is available at the end of the article
}

the CSF. It is well recognized that the CP's principal function is the production of CSF [1]. However, it is also known that the $\mathrm{CP}$ participates in other key functions, namely the transport of nutrients into the brain and the removal of metabolic products out of the brain [2]. This seems to be of relevance in diseases such as Alzheimer's, where CP proteins in the apical membrane participate in the clearance of the amyloid beta peptide, alone or bound to CSF carrier proteins [3-5]. In addition, the $\mathrm{CP}$ is able to respond to challenges by displaying additional functions not present under basal conditions. It has been recently shown that the CP specifically responds differently to peripheral inflammation, depending on whether the inflammation is acute or sustained, by secreting several immuno modulators [6-9]. Moreover, in conditions of inflammation, the $\mathrm{CP}$ seems to be important in the regulation of brain iron

\section{() Biomed Central}


homeostasis [10,11]. The CP is also one of the first places of entry for the immune cells during neurological disorders, as shown in the experimental autoimmune encephalomyelitis model of multiple sclerosis [12].

Although a vast number of $\mathrm{CP}$ mRNA/proteins have been previously described in the literature, discussion of the full basal transcriptome is still lacking. The data herein presented supports the view that the $\mathrm{CP}$ is more than a barrier that restricts the passage of molecules and cells in and out of the brain, but rather a tissue that should be better studied as an active participant in brain homeostasis in both physiological and pathological conditions.

\section{Methods}

All experiments and procedures followed the European Community Council Directive 86/09/EEC guidelines for the care and handling of laboratory animals and were approved by the Life and Health Sciences Research Institute's ethical committee and by the Portuguese Veterinary Authorities ("Direcção Geral de Veterinária" process reference number 520/000/000/2006).

\section{Animals}

All experiments were conducted using 8- to 9-week old C57BL/6 male mice (Charles River, Barcelona, Spain). Animals were maintained under $12 \mathrm{~h}$ light/dark cycles at $22.5^{\circ} \mathrm{C}$ and $55 \%$ humidity and fed with regular rodent chow and tap water ad libitum. In order to reduce the stress-induced changes in the hypothalamus-pituitary axis, animals were handled for 1 week prior to the start of the experiment. Mice were anesthetized with intraperitoneal injection of ketamine hydrochloride $(150 \mathrm{mg} / \mathrm{Kg})$ plus medetomidine $(0.3 \mathrm{mg} / \mathrm{Kg})$ and transcardially perfused with cold saline. The CPs were rapidly removed, stored in RNA later (Ambion, Austin, TX, USA) and kept at $-80^{\circ} \mathrm{C}$. $\mathrm{CP}$ isolation was made under conventional light microscopy (SZX7, Olympus, Hamburg, Germany). Three pools of $\mathrm{CP}$ (each containing tissue removed from the 4 ventricles of 3 mice) were prepared at the time of sacrifice. While most of the cells are CP epithelial cells, the tissue sampled may also contain stroma cells (e.g. endothelial cells and pericytes). These animals correspond to those used as controls (basal transcriptome) in Marques et al. [8].

\section{Microarray experimental design and data analysis}

Total RNA was isolated with Trizol (Invitrogen, Carlsbad, CA, USA) following manufacturer's instructions. After quality assessment using the Agilent Bioanalyzer (Agilent Technologies, CA, USA), 100 ng of total RNA from each pool was amplified and labelled with Illumina TotalPrep RNA Amplification Kit (Illumina Inc., San Diego, CA, USA). The labelled cRNA was then hybridized in a total of two Illumina Whole-genome Mouseref- 8 expression
Beadchips (Illumina Inc.), following the recommended protocol, each containing 8 arrays, comprising a total of 24,049 well-annotated RefSeq transcripts per beadchip.

After scanning, raw data from BeadStudio software (Illumina Inc.) was read into R/Bioconductor and normalized using quantile normalization. A linear model was applied to the normalized data using Limma package in R/Bioconductor [13]. A contrast analysis was applied and differentially expressed genes were selected using a Bayesian approach with a false discovery rate of $5 \%$. All data is Minimum Information About a Microarray Experiment (MIAME)-compliant and the raw data has been deposited in the GEO database (accession: GSE23714). In order to analyze and compare the CP transcriptome with transcriptomes from other tissues, the initial list containing the 24,049 CP genes was filtered: the repeated genes present in the array platform were excluded and the genes with a Coefficient of Variation $(\mathrm{CV}) \geq 30$ across the 3 replicates were also removed. The final gene list included 18,160 genes. For the analysis, normalized genes were categorized using the Gene Set Analysis Toolkit from WebGestalt (http://bioinfo.vanderbilt.edu/webgestalt/ [14]) or the Ingenuity tools (Redwood City, CA, USA). Enrichment analysis was performed using DAVID (http://david.abcc. ncifcrf.gov/ [15]) and Ingenuity (http://www.ingenuity.com [16]) software. For comparison with other transcriptomes, two studies of the GEO database were used: one containing 61 mouse different tissues in physiological conditions (GDS592) [17], and another containing the transcriptome of the PECAM-1+ blood-brain barrier (BBB) microvascular endothelial cells (ppMBMECs) (GSE14375) [18]. From each gene list the repeated genes and the genes with $\mathrm{CV} \geq$ 30 were removed. After these corrections, a list containing the genes common in the three studies was created, analyzed with the Limma package to normalize data between arrays and, subsequently, a cluster was constructed using the Multiexperiment Viewer program.

The GEO database has data on two other CP studies. One focuses on the differential $\mathrm{CP}$ expression between B10.pl WT and B10.PL RAG-/- mice (GSE11443); the other evaluates the gene expression profile of 24 neural tissues (including the CP from the $4^{\text {th }}$ ventricle) and 10 other body tissues/organs (GSE3594). We compared our transcriptome with the control transcriptome of each of these two other studies. For these comparisons, we selected the common genes excluding those presenting a $C V \geq 30$. After these corrections, data between arrays was normalized with the Limma package, followed by a comparison of the expression level for each gene.

\section{Confirmation of the array data by RT-qPCR}

In order to validate the array data, 20 genes within the lower range of expression (6.1-7.0) were analyzed in an independent set of CP samples. The selected genes 
were: inducible T-cell co-stimulator, myotubularinrelated protein 7 , retinal pigment epithelium 65 , dual oxidase 2 , arginine vasopressin receptor $1 \mathrm{~B}$, glutathione peroxidase 5, nestin, epithelial mitogen, interleukin 6 , neuropeptide Y, matrix metalloproteinase 9, glucagon receptor, claudin 15 , ceruloplasmin, lipocalin 2, CD14 antigen, vav 1 oncogene, retinoic acid receptor gamma, interferon regulatory factor 1 and toll-like receptor 4 . Gene abbreviations and gene names are specified in accordance with the HUGO Gene Nomenclature Committee at http://www.genenames.org/ [19]. Total RNA was isolated from five pools of CP using Trizol reagent (Invitrogen), and $500 \mathrm{ng}$ amplified using the Superscript RNA amplification system (Invitrogen) according to the manufacturer's instructions. CP RNA was reverse transcribed using random primers of the Superscript Firststrand Synthesis system for RT-PCR (Invitrogen).

The oligonucleotide primers (sequences available upon request) were designed using the Primer3 software on the basis of the GenBank sequences. Real-time PCR reactions, using equal amounts of total RNA from each sample, were performed on a CFX $96^{\mathrm{TM}}$ real-time system instrument (Bio-Rad Laboratories, Hercules, CA, USA) using QuantiTect SYBR Green RT-PCR reagent kit (Qiagen, Hamburg, Germany). Product fluorescence was detected at the end of the elongation cycle. All melting curves exhibited a single sharp peak at a temperature characteristic of the primers used.

\section{Construction of biological function networks}

In order to identify major functions of the $\mathrm{CP}$, networks based on the genes displaying higher expression (threshold 13 in the array) were constructed. Analysis yielded 59 genes that were subsequently analysed in the FunNet (http://www.funnet.info [20]) for GO Molecular Function and Kyoto Encyclopedia of Genes and Genomes (KEGG) functional networks. This program clusters the genes depending on their biological function, creating nodes corresponding to those that are most enriched. The relation between every pair of genes is calculated using the signal expression of the genes on the corresponding biological functions.

\section{Results}

\section{$\mathrm{CP}$ transcriptome characterization}

From the complete array analysis, we found that the majority of genes in the $\mathrm{CP}$ transcriptome are expressed at very low levels. From a range of expression levels that varied from 6.1-15.0, (log2-transformed absolute expression levels), $81 \%$ of the genes were expressed between the 6.1-8.3 level; $15 \%$ were expressed at levels between 8.3-10.5; $4 \%$ were expressed with the level between 10.512.8 and only $0.4 \%$ of the genes were highly expressed and presented an expression level between 12.8-15.0
(Figure 1). All the genes detected and their relative expression levels are presented as Additional File 1 (Expression levels of the genes transcribed in the basal choroid plexus) and deposited in the GEO database GSE23714.

Twenty genes, with expression levels ranging from 6.1 to 7.0, were further analyzed by RT-qPCR in CP collected from an independent group of animals. This analysis confirmed the relative expression of these genes (data not shown), and indicated that genes with an expression level of 6.1, the lowest level in the array, are detectable by RT-qPCR. Altogether, it was inferred from the analysis that the array data represented genes expressed under basal physiological conditions.

Table 1 lists the fifty-nine most highly expressed genes (threshold 13.0 in the array). Analysis of the genes displaying expression levels above 13 were clustered in particular functional networks (Figure 2). Analysis of gene function networks identified 3 modules of interaction for the 59 mostly expressed genes. One included genes participating in pathways such as regulation of the tissue remodelling, fibril organization, negative regulation of processes such as extracellular matrix disassembly and blood vessel remodelling. Another, included genes of iron homeostasis, carbohydrate and lipid metabolism. A third module included genes involved in free radical homeostasis, regulation of the inflammatory response, mitochondrial metabolism and RNA processing. A table containing the various genes in each module is provided as Additional File 2 (Functional networks of the most highly-expressed genes in the choroid plexus).

Figure 3 identifies the molecular pathways encompassing genes with an expression level between 6.1-12.8. Of

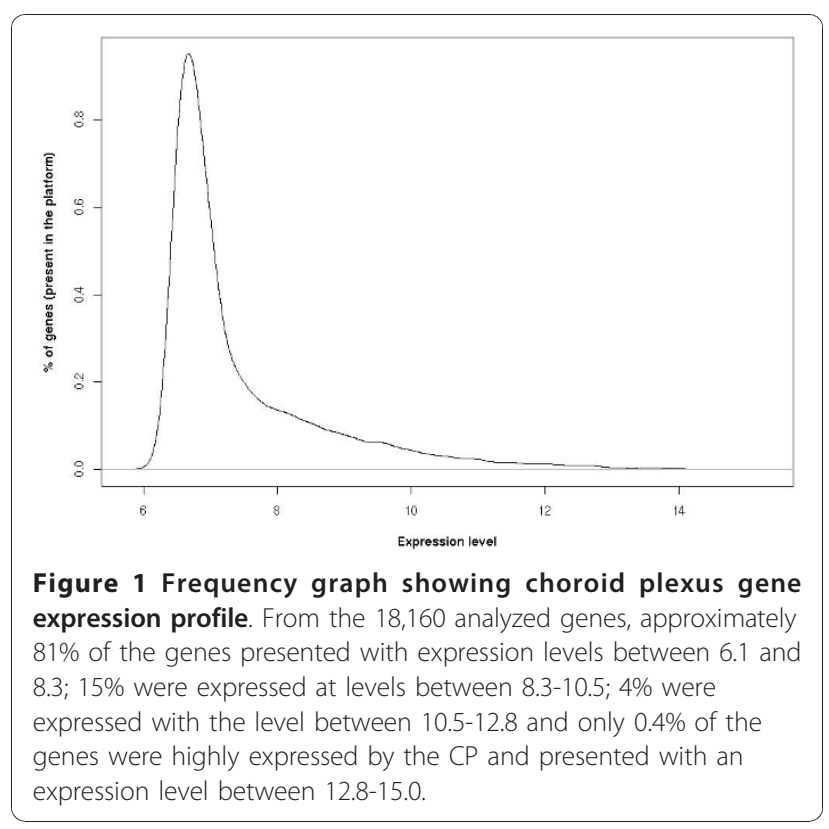


Table 1 Most highly expressed genes found in the choroid plexus in normal physiological conditions

\begin{tabular}{|c|c|c|}
\hline Symbol & Definition & E.value \\
\hline Ubb & ubiquitin B & 15.0 \\
\hline $\operatorname{lgf2}$ & insulin-like growth factor 2 & 14.6 \\
\hline Rpl41 & ribosomal protein $L 41$ & 14.5 \\
\hline Cox4i1 & cytochrome c oxidase subunit IV isoform 1 & 14.1 \\
\hline Clu & clusterin & 14.1 \\
\hline Psap & prosaposin & 14.0 \\
\hline Chchd10 & coiled-coil-helix-coiled-coil-helix domain containing 10 & 14.0 \\
\hline Arl6ip1 & ADP-ribosylation factor-like 6 interacting protein 1 & 13.9 \\
\hline Atp5b & ATP synthase, $\mathrm{H}+$ transporting mitochondrial F1 complex. beta subunit & 13.9 \\
\hline Cst3 & cystatin C & 13.9 \\
\hline Clic6 & chloride intracellular channel 6 & 13.8 \\
\hline Ttr & transthyretin & 13.8 \\
\hline 1500015010Rik & RIKEN cDNA 1500015010 gene & 13.7 \\
\hline Grim19 & genes associated with retinoid-IFN-induced mortality 19 & 13.7 \\
\hline Cox8a & cytochrome c oxidase, subunit VIlla & 13.7 \\
\hline Gapd & glyceraldehyde-3-phosphate dehydrogenase & 13.7 \\
\hline Atp5h & ATP synthase. $\mathrm{H}+$ transporting, mitochondrial F0 complex. subunit $d$ & 13.7 \\
\hline Uba52 & ubiquitin A-52 residue ribosomal protein fusion product 1 & 13.6 \\
\hline Uqcrh & ubiquinol-cytochrome c reductase hinge protein & 13.6 \\
\hline $\mathrm{Ckb}$ & creatine kinase. brain & 13.6 \\
\hline Cox6a1 & cytochrome c oxidase. subunit VI a, polypeptide 1 & 13.6 \\
\hline Gpx4 & glutathione peroxidase 4 & 13.6 \\
\hline Rps14 & ribosomal protein S14 & 13.6 \\
\hline Ppia & peptidylprolyl isomerase A & 13.6 \\
\hline Rplp1 & ribosomal protein. large, P1 & 13.6 \\
\hline Aldh2 & aldehyde dehydrogenase 2 , mitochondrial & 13.6 \\
\hline Rnaset2 & ribonuclease $\mathrm{T} 2$ & 13.5 \\
\hline Rps27a & ribosomal protein S27a & 13.5 \\
\hline Rps29 & ribosomal protein S29 & 13.5 \\
\hline Dbi & diazepam binding inhibitor & 13.5 \\
\hline Atp5j2 & ATP synthase, $\mathrm{H}+$ transporting. mitochondrial F0 complex, subunit f. isoform 2 & 13.5 \\
\hline Grina & glutamate receptor, ionotropic. N-methyl D-asparate-associated protein 1 (glutamate binding) & 13.5 \\
\hline 1110020P15Rik & RIKEN cDNA $1110020 P 15$ gene & 13.5 \\
\hline Atp1a1 & ATPase, $\mathrm{Na}+/ \mathrm{K}+$ transporting. alpha 1 polypeptide & 13.5 \\
\hline Fth1 & ferritin heavy chain 1 & 13.4 \\
\hline Ptgds & prostaglandin D2 synthase (brain) & 13.4 \\
\hline Atp5g3 & ATP synthase. $\mathrm{H}+$ transporting, mitochondrial F0 complex. subunit c (subunit 9), isoform 3 & 13.4 \\
\hline Cd81 & Cd81 antigen & 13.4 \\
\hline Ndufa1 & NADH dehydrogenase (ubiquinone) 1 alpha subcomplex. 1 & 13.3 \\
\hline Atp1b1 & ATPase. $\mathrm{Na}+/ \mathrm{K}+$ transporting, beta 1 polypeptide & 13.2 \\
\hline Cd63 & Cd63 antigen & 13.2 \\
\hline Cox5b & cytochrome c oxidase. subunit Vb & 13.2 \\
\hline Ubc & ubiquitin C & 13.2 \\
\hline Sostdc1 & sclerostin domain containing 1 & 13.2 \\
\hline Rps20 & ribosomal protein $\mathrm{S} 20$ & 13.2 \\
\hline Ubl5 & ubiquitin-like 5 & 13.1 \\
\hline Aplp2 & amyloid beta (A4) precursor-like protein 2 & 13.1 \\
\hline Scd2 & stearoyl-Coenzyme A desaturase 2 & 13.1 \\
\hline Rbp1 & retinol binding protein 1. cellular & 13.1 \\
\hline Ndufa4 & NADH dehydrogenase (ubiquinone) 1 alpha subcomplex, 4 & 13.1 \\
\hline Rpl3 & ribosomal protein L3 & 13.1 \\
\hline
\end{tabular}


Table 1 Most highly expressed genes found in the choroid plexus in normal physiological conditions (Continued)

\begin{tabular}{lll}
\hline 2010107E04Rik & RIKEN CDNA 2010107E04 gene & 13.1 \\
Cryab & crystallin, alpha B & 13.0 \\
Vdac1 & voltage-dependent anion channel 1 & 13.0 \\
Car12 & carbonic anyhydrase 12 & 13.0 \\
Rpl35 & ribosomal protein L35 & 13.0 \\
Cox7c & cytochrome c oxidase. subunit VIlc & 13.0 \\
Rps21 & ribosomal protein S21 & 13.0 \\
Ldh2 & lactate dehydrogenase 2, B chain & 13.0 \\
\hline
\end{tabular}

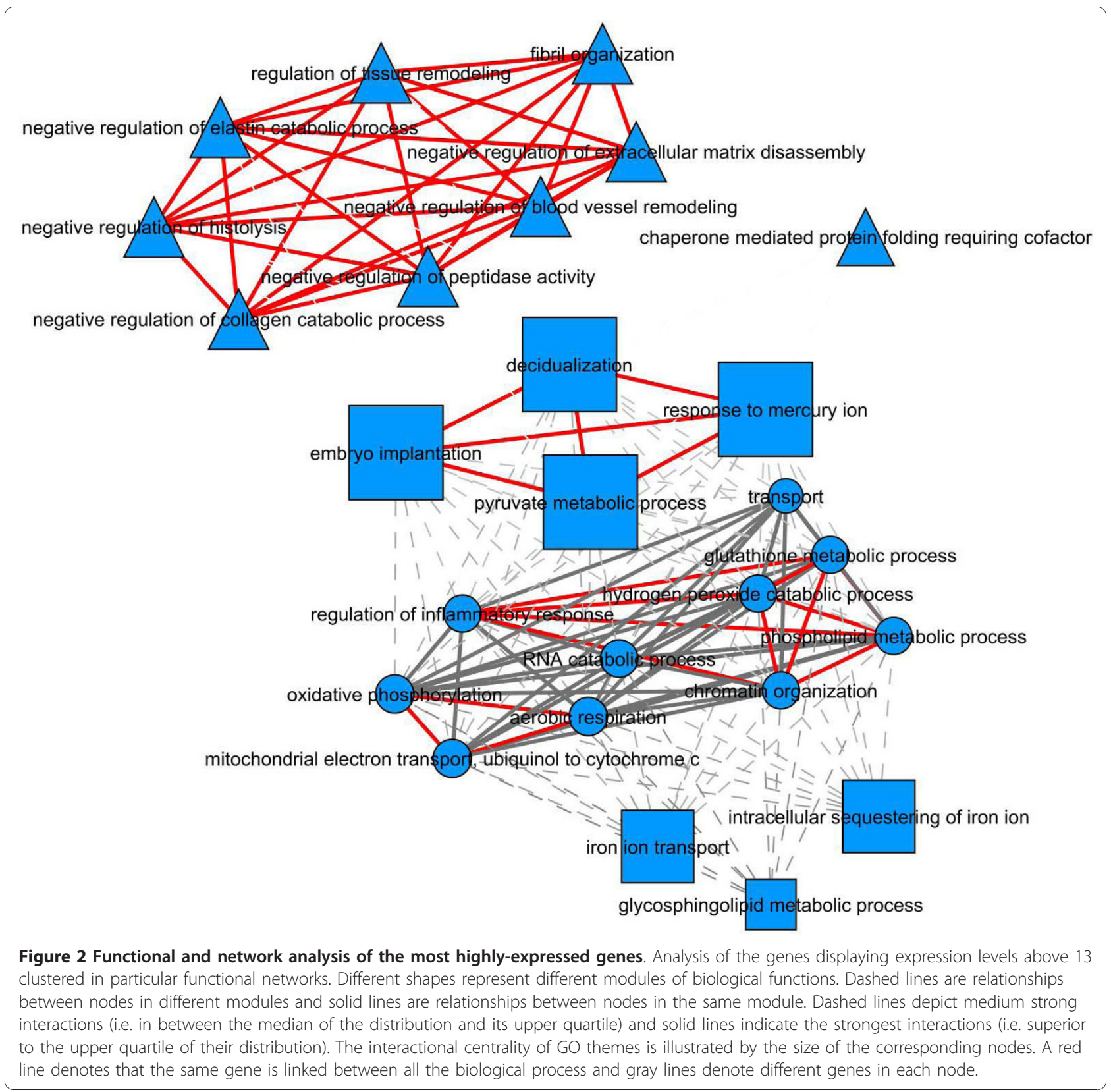


note, is the number of genes clustered in classes related to oxidative phosphorylation and ribosomal function, which is in agreement with the high secretory capacity of the CP. Interestingly, adhesion and regulation of actin cytoskeleton are also highly expressed, probably reflecting the barrier functions. In addition, it highlights genes belonging to various signalling pathways.
Classes of genes expressed in the $\mathrm{CP}$ and their functions

The CP has traditionally been viewed as a tissue that provides the brain with secreted CSF, which through bulk flow, supplies the brain with some nutrients such as vitamin $\mathrm{C}$, amino acids, nucleosides and transport proteins such as transthyretin. The CP is also known to facilitate the removal of products of brain metabolism

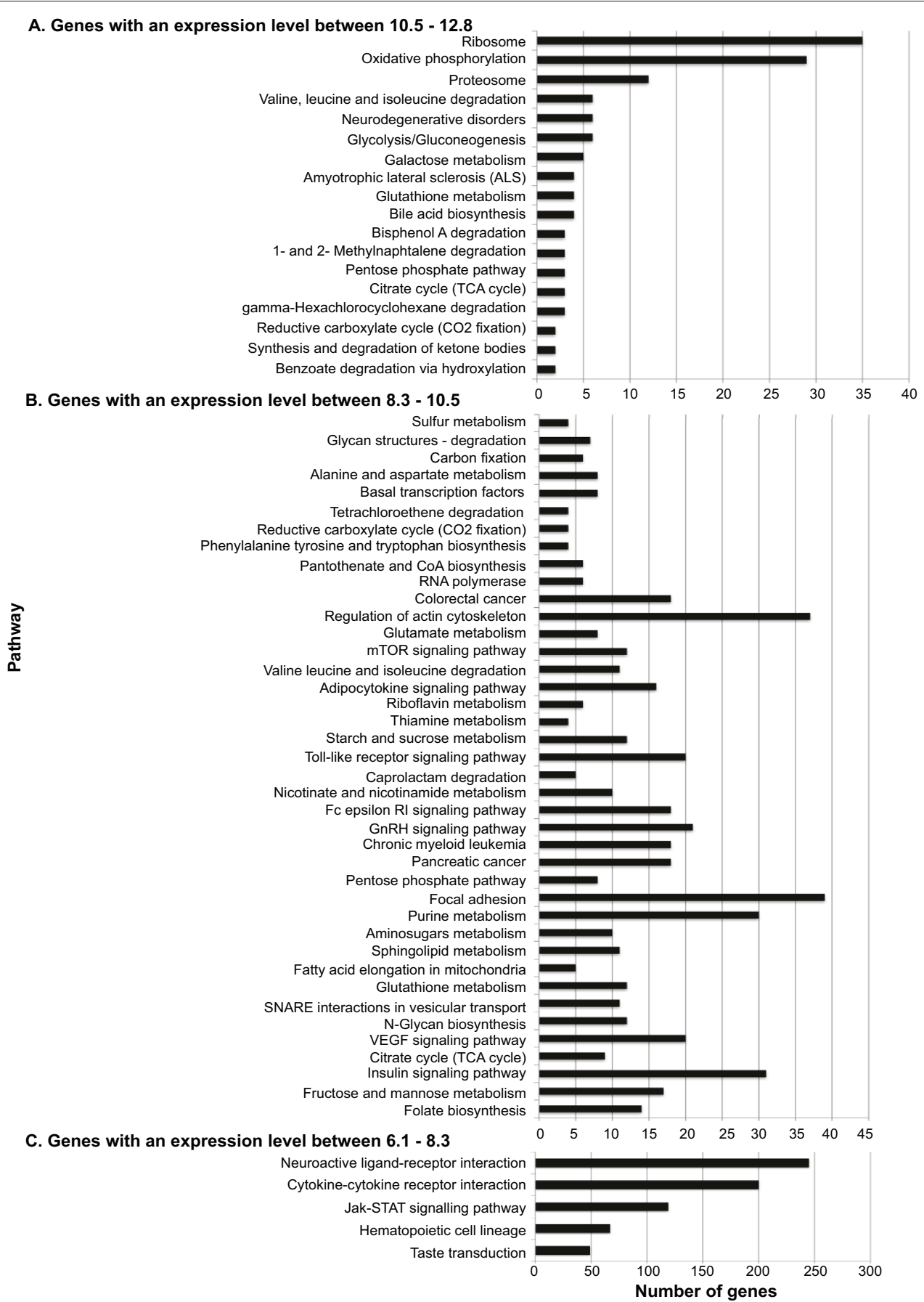

Figure 3 Numbers of genes in different metabolic pathways expressed in the choroid plexus in normal physiological conditions Genes that have expression levels from 6.1-8.3 (A); from 8.3-10.5 (B) and from 10.5-12.8 (C). 
such as free iodine, glutamate and amyloid beta peptide. On the other hand, the $\mathrm{CP}$ provides a barrier that restricts the passage of molecules and cells into and out of the brain. The present study provides solid evidence to support this view. In agreement we found that the $\mathrm{CP}$ transcriptome includes genes encoding for the tight junctions proteins (Figure 4A), for transporters namely for monocarboxylic acid (Slc16a9), glucose (Slc2a1), vitamin C (Slc23a2), thiamine (Slc19a2), zinc (such as Slc39a1) (Figure 4B) and for receptors of various classes of neurotransmitters such as dopamine, serotonin, opioids, cannabinoids, glutamate, histamine and GABA and also receptors for folate, benzodiazepine, estrogen and growth hormone (Figure $4 \mathrm{C}$ ). Of notice, with

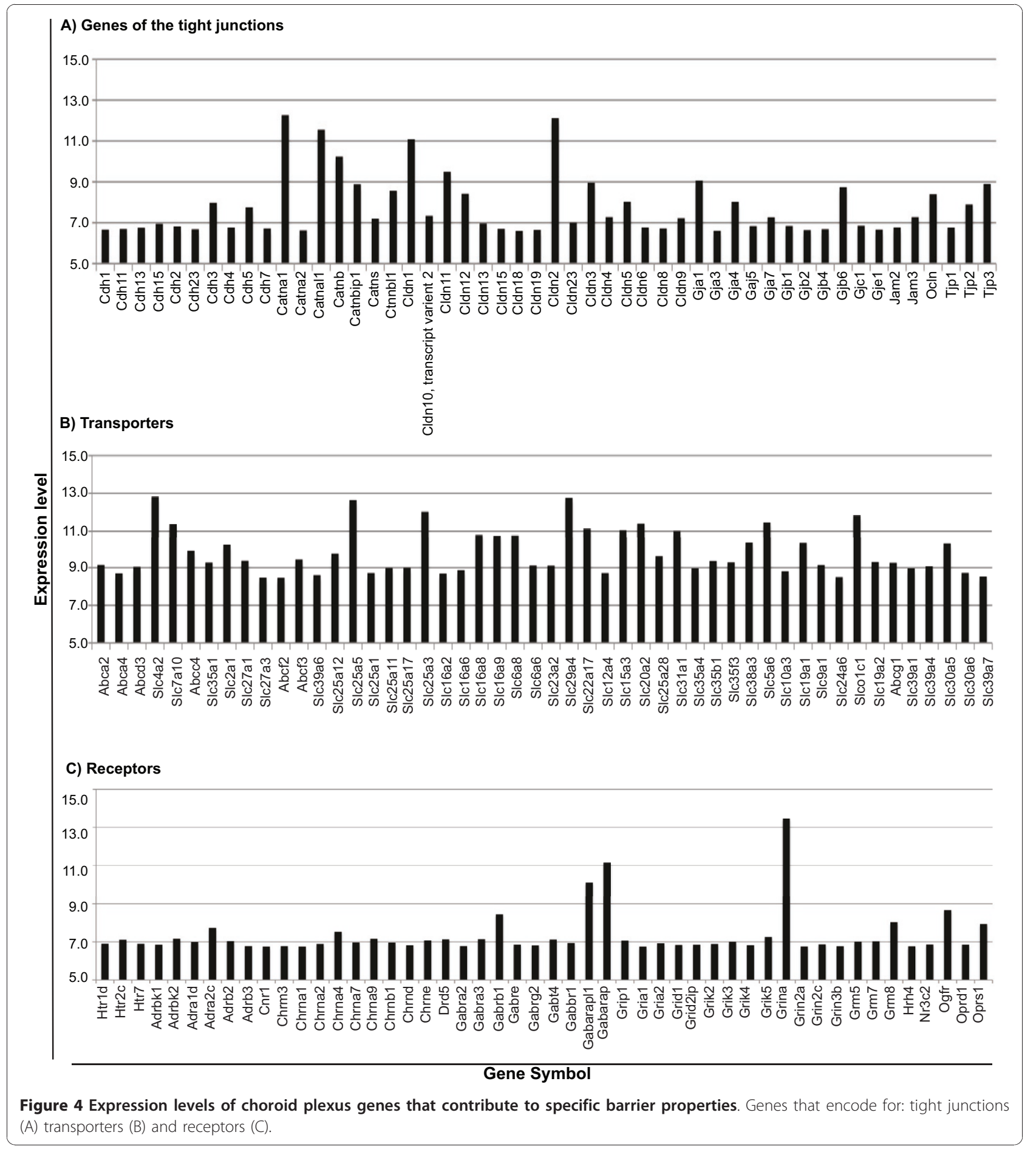


respect to transporters, the $\mathrm{CP}$ highly expressed both the $\mathrm{ABC}$ (all 7 distinct sub-families: $\mathrm{ABC1}$, MDR/TAP, MRP, ALD, OABP, GCN20, White) and the Slc families (Figure 4B). In addition to the MDR/TAP and MRP, the basal transcriptome also revealed the presence of the solute carrier organic anion families (OAT and OATP) of efflux transporters. Finally, the sodium-dependent system for the excitatory acidic amino acids, e.g., glutamate and aspartate, was also expressed in the CP and suggests a mechanism for net removal of potentially neurotoxic amino acids from the brain. The $\mathrm{CP}$ also expressed different genes that encode transporters for glucose, fatty acid, monocarboxylic acid, nucleoside, glycerol, cationic amino acid transporter $\mathrm{y}^{+}$system, iodide and neurotransmitter transporters (Figure 4B).
We chose to further highlight genes encoding for axonal guidance (Figure 5A), and for growth factors (Figure 5B) given their relevance for brain development and also for adult neurogenesis, as will be discussed later. These include insulin-like growth factor 2, transforming growth factor beta 2, vascular endothelial growth factor $\mathrm{A}$ and $\mathrm{B}$, brain derived neurotrophic factor, ciliary neurotrophic factor transcript variant 2, fibroblast growth factors, betacellulin epidermal growth factor family member and its receptor ErbB-4, neuregulin 1, 3 and 4, amphiregulin, epiregulin and several members of the Wnt family (Additional File 1). With respect to axonal guidance, the CP basal transcriptome includes the four conserved families of guidance cues shown to have prominent developmental effects: netrins, semaphorins,

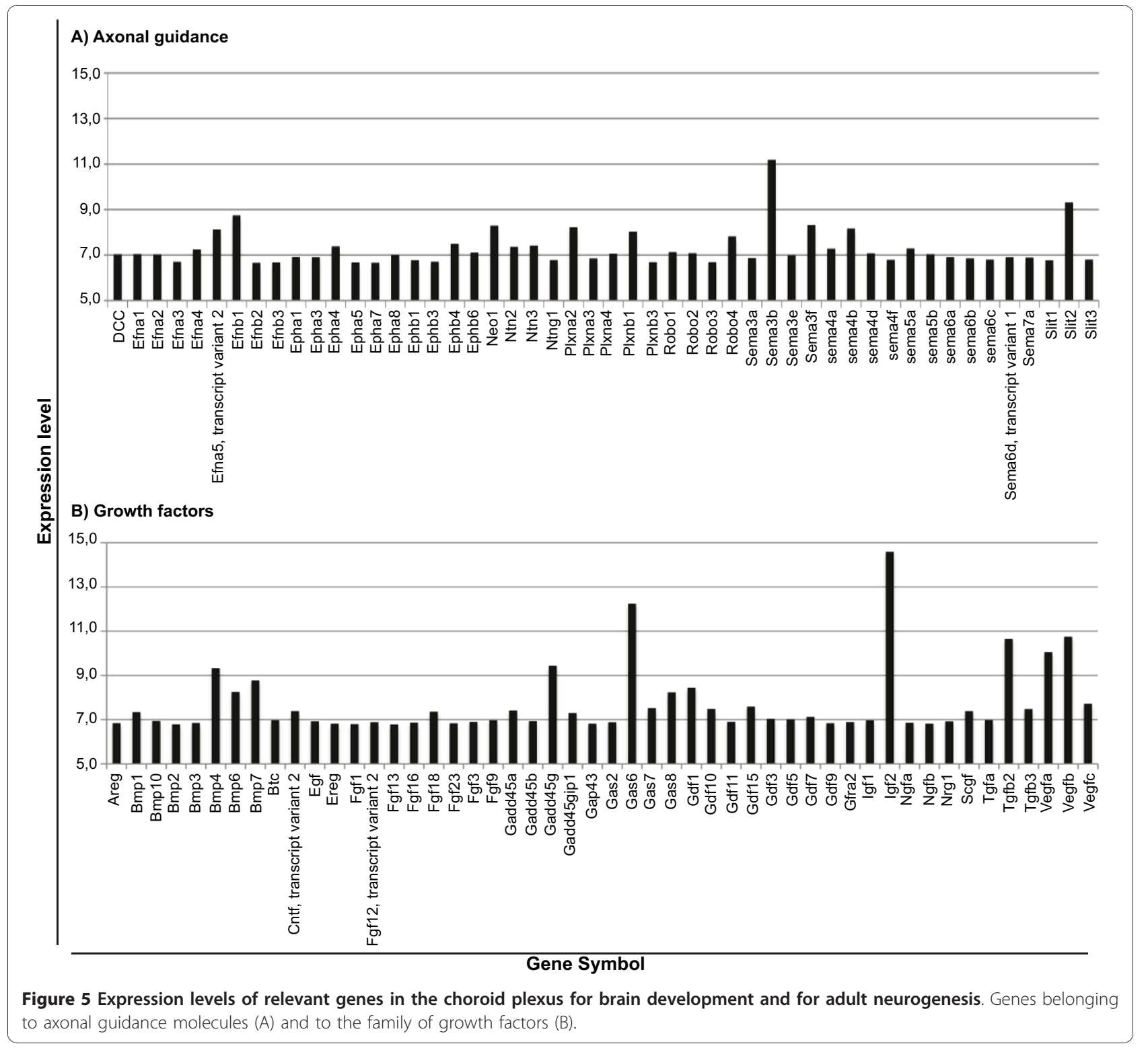


SLIT and ephrins, as well as their respective receptors. Not surprisingly, among the most highly expressed genes are those encoding for major CSF proteins, such as transthyretin, prostaglandin D2 synthase, transferrin, insulin-like growth factor 2, clusterin (apolipoprotein J) and cystatin $\mathrm{C}$ (Table 1 ).

\section{CP transcriptome comparison with other mouse tissue transcriptomes}

From the 62 tissues analysed, the CP transcriptome correlated with the transcriptome of the $\mathrm{BBB}$ endothelial cells more closely than with tissues from other brain regions or other peripheral tissues (data not shown).

We compared our CP array data with two others that are deposited in the GEO database, the GSE11443 and GSE3594. We observed that the correlation between our CP data was approximately $56 \%$ when compared to GSE11443 and 57\% when compared to GSE3594. To test if the correlation we found between our study and the two others was acceptable, we also compared the correlation between the two other studies and found a correlation of $63 \%$. Interestingly a similar correlation was found when the liver transcriptome from different studies was compared, which in this case had a correlation of $53 \%$. Since the mostly expressed genes are those that best characterize a tissue, we also analysed if the fifty most highly-expressed genes in our study corresponded to the same fifty most highly-expressed genes in the two other CP studies. We observed that 34 and 20, from the GSE11443 and GSE3594 respectively, are common between the studies. Among the mostly expressed genes in these 3 studies are the genes encoding for Arl6ip1, Ckb, Cox6a1, Cst3, Igf2, Ptgds, Rbp1, Rpl41, Slc4a2, Ttr and Ubc.

\section{Discussion}

Here we analysed and characterized the mouse CP transcriptome under normal basal physiological conditions. Unravelling the transcriptome signature of a tissue is a valuable tool for better understanding its functions in health and in disease. In this particular case, as part of the barriers that separate the brain from the periphery, it has become clear that the CP is more than abstacle for molecule and cell trafficking into and out of the brain; it is an active participant in brain homeostasis.

Not surprisingly, we confirmed that among the genes most highly expressed were those encoding for proteins that are secreted into the CSF, such as transthyretin and transferrin. These are carriers for the ligands thyroxine and iron respectively, which are relevant for proper brain function during development and throughout adulthood. While CP transthyretin in itself does not seem essential for thyroid hormone to reach and distribute within the brain parenchyma [21], the available data does not exclude a role for the $\mathrm{CP}$ in thyroid hormone homeostasis. In fact, the thyroid gland is the sole site recognized for thyroid hormone synthesis. However, when the thyroid gland is ablated, it takes several months for the tissues to be depleted of thyroid hormones [22,23], which raises the possibility of extrathyroidal sites for thyroid hormone synthesis. The CP could be such a place, not only because it expresses the sodium-iodine symporter [24], which uptakes iodine against a concentration gradient, but also several peroxidases (glutathione peroxidase $4,1,3,7$, myeloperoxidase, thyroid peroxidase) as we now show in this study. Future investigation should address whether these peroxidases have the same ability as thyroid peroxidase in the thyroid to oxidise iodine and incorporate it into thyroglobulin (also present in the basal CP transcriptome) or other tyrosine containing protein.

Analysis of the basal transcriptome revealed genes involved in iron homeostasis such as transferrin; however, it is notable that participation in brain iron homeostasis seems to be particularly relevant in response to stimuli such as peripheral inflammation [11]. These observations highlight the ability of the CP to respond/ adapt to physiological and disease states.

Several genes were shown to be expressed in the CP for the first time. These include those encoding for the opioid receptors, for which we found mRNA for all members of the family, the opioid receptor sigma 1 being the most highly expressed. Other studies, using autoradiographic techniques with selective ligands, failed to detect the $\mathrm{mu}$ and delta opioid receptors in rat $\mathrm{CP}$ brain $[25,26]$, probably due to the lower sensitivity of the methodologies used. The present study also confirmed the presence of other neurotransmitter receptors previously described in the CP [27], such as the dopamine receptors. Again, this emphasizes an active role of the $\mathrm{CP}$ as a potential site for modulating response to neurotransmitters, which should be further investigated in the context of mood disorders.

We consider it of interest that the adult CP expresses genes encoding for several growth factors and axonal guidance molecules. While semaphorin 3f [2] and Slits 2 and $3[28,29]$ were previously described in the developing $\mathrm{CP}$, we described here for the first time the expression of various netrins and ephrins. The present study also adds to previously described growth and trophic factors [30,31], such as insulin-like growth factor II [32], glial cell line-derived neurotrophic factor [33], transforming growth factor alpha [34], fibroblast growth factor 2 [35] and vascular endothelial growth factor [36]. The expression of genes encoding for several members of the FGF family (those with higher expression value being FGF 18, 9 and 3), neuregulin, betacellulin and epiregulin should be highlighted. Betacellulin and its receptor ErbB-4 are highly expressed in neurons, implying important roles in neuronal cell functions; and 
inducing ErbB-4 activation promotes neurite outgrowth in PC12 cells [37]. Numerous genetic modelling studies in mice demonstrate that neuregulin 1 signalling is important in the development of normal neuronal connectivity. Of particular interest to this discussion are findings linking deficits in neuregulin 1-ErbB4 signalling to perturbations of synaptic transmission, myelination, and the survival of particular sets of neurons and glia [38]. It will be of interest to next investigate the participation of the $\mathrm{CP}$ in demyelinating diseases.

The CP expression of growth factors is also relevant in the context of neurogenesis. The subventricular zone is, together with the hippocampus, a site of adult neurogenesis. We showed here that the genes encoding for molecules that modulate adult neural stem cell proliferation and fate are transcribed in the $\mathrm{CP}$. It is surprising that few studies so far have considered the $\mathrm{CP}$ as a relevant source of such mediators. The present study draws attention to the CP, as a modulator of the CSF composition, in the distribution of key molecules for neuronal differentiation.

Finally, the data we presented here confirms that the $\mathrm{CP}$ transcriptome displays a specific signature, different from all other tissues for which transcriptome data is available [39]. While one could anticipate resemblance with other secretory epithelia, such as the lung, the closest similarity is with that of the PECAM-1+ BBB microvascular endothelial cells [18]. This most likely reflects the fact that both have a barrier function, mediated by tight junctions; participate in the transport of molecules in and out of the brain, through several transporters and receptors; and in detoxifying processes, through several enzymes $[31,40]$. Despite these similarities, there are striking differences in gene expression between these two barriers, such as is the case for carbonic anhydrase 12, which is involved in CSF production (13.0 versus 4.4).

\section{Conclusions}

In summary, the present work reveals that the $\mathrm{CP}$ is a site of active expression of several molecular pathways of relevance for brain homeostasis. Understanding its involvement in various physiological and disease states may provide novel clues to disease mechanisms, and also unravel novel targets for therapies against diseases of the central nervous system.

\section{Additional material}

Additional file 1: Expression levels of the genes transcribed in the basal choroid plexus. The table lists the expression levels of all genes expressed in the basal CP transcriptome, after normalization as specified in the Methods section.

Additional file 2: Functional networks of the most highly-expressed genes in the choroid plexus. The 59 most highly expressed genes were grouped in a 3-modular theme proximity network. The genes belonging to each module are specified.

List of abbreviations

BBB: blood-brain barrier; CP: choroid plexus; CSF: cerebrospinal fluid; CV: coefficient of variation; KEGG: Kyoto Encyclopedia of Genes and Genomes; MIAME: Minimum Information About a Microarray Experiment; ppMBMECs: PECAM-1+ blood-brain barrier microvascular endothelial cells; RT-qPCR: real time quantitative PCR.

\section{Acknowledgements}

This work was supported by a grant from The Dana Foundation (USA); Marques $F$ is recipient of postdoctoral fellowship from Fundação para a Ciência e Tecnologia (Portugal).

\section{Author details}

${ }^{1}$ Life and Health Sciences Research Institute (ICVS), School of Health Sciences, University of Minho, Campus Gualtar, 4710-057 Braga, Portugal. ${ }^{2}$ Program in Neurogenetics, Department of Neurology, David Geffen School of Medicine-UCLA, Los Angeles, CA, 90095-1761, USA. ${ }^{3}$ Hospital A. C. Camargo, São Paulo, SP, 01509-010, Brazil.

\section{Authors' contributions}

FM: conceived the study, participated in its design, preformed the experiments and collected, analysed and interpreted the data. JCS: conceived the study and participated in its design. GC: collected, analysed and interpreted the data. FG, RP, HB, and DG analysed and interpreted the data. NS: Conceived the study, participated in its design and discussed the findings. MCN: Conceived the study, participated in its design and discussed the findings. JAP: Conceived the study, participated in its design, discussed the findings and coordinated the study.

All authors have read and approved the final version of the manuscript.

\section{Competing interests}

The authors declare that they have no competing interests.

Received: 11 November 2010 Accepted: 18 January 2011

Published: 18 January 2011

\section{References}

1. Speake T, Whitwell C, Kajita H, Majid A, Brown PD: Mechanisms of CSF secretion by the choroid plexus. Microsc Res Tech 2001, 52:49-59.

2. Redzic ZB, Preston JE, Duncan JA, Chodobski A, Szmydynger-Chodobska J: The choroid plexus-cerebrospinal fluid system: from development to aging. Curr Top Dev Biol 2005, 71:1-52.

3. Zlokovic BV, Martel CL, Matsubara E, McComb JG, Zheng G, McCluskey RT, Frangione B, Ghiso J: Glycoprotein 330/megalin: probable role in receptor-mediated transport of apolipoprotein $\mathrm{J}$ alone and in a complex with Alzheimer disease amyloid beta at the blood-brain and blood-cerebrospinal fluid barriers. Proc Natl Acad Sci USA 1996, 93:4229-4234.

4. Zlokovic BV: Clearing amyloid through the blood-brain barrier. Neurochem 2004, 89:807-811.

5. Sousa JC, Cardoso I, Marques F, Saraiva MJ, Palha JA: Transthyretin and Alzheimer's disease: where in the brain? Neurobiol Aging 2007, 28:713-718.

6. Konsman JP, Vigues S, Mackerlova L, Bristow A, Blomqvist A: Rat brain vascular distribution of interleukin-1 type-1 receptor immunoreactivity: relationship to patterns of inducible cyclooxygenase expression by peripheral inflammatory stimuli. J Comp Neurol 2004, 472:113-129.

7. Marques F, Sousa JC, Correia-Neves M, Oliveira P, Sousa N, Palha JA: The choroid plexus response to peripheral inflammatory stimulus. Neuroscience 2007, 144:424-430.

8. Marques F, Sousa JC, Coppola G, Falcao AM, Rodrigues AJ, Geschwind DH, Sousa N, Correia-Neves M, Palha JA: Kinetic profile of the transcriptome changes induced in the choroid plexus by peripheral inflammation. $J$ Cereb Blood Flow Metab 2009, 29:921-932.

9. Mitchell K, Yang HY, Berk JD, Tran JH, ladarola MJ: Monocyte chemoattractant protein-1 in the choroid plexus: a potential link 
between vascular pro-inflammatory mediators and the CNS during peripheral tissue inflammation. Neuroscience 2009, 158:885-895.

10. Marques F, Rodrigues AJ, Sousa JC, Coppola G, Geschwind DH, Sousa N Correia-Neves M, Palha JA: Lipocalin 2 is a choroid plexus acute-phase protein. J Cereb Blood Flow Metab 2008, 28:450-455.

11. Marques F, Falcao AM, Sousa JC, Coppola G, Geschwind D, Sousa N, Correia-Neves M, Palha JA: Altered iron metabolism is part of the choroid plexus response to peripheral inflammation. Endocrinology 2009, 150:2822-2828.

12. Reboldi A, Coisne C, Baumjohann D, Benvenuto F, Bottinelli D, Lira S, Uccelli A, Lanzavecchia A, Engelhardt B, Sallusto F: C-C chemokine receptor 6-regulated entry of $T(H)-17$ cells into the CNS through the choroid plexus is required for the initiation of EAE. Nat Immunol 2009 10(5):514-23, Epub 2009 Mar 22.

13. Gentleman RC, Carey VJ, Bates DM, Bolstad B, Dettling M, Dudoit S, Ellis B, Gautier L, Ge Y, Gentry J, Hornik K, Hothorn T, Huber W, lacus S, Irizarry R, Leisch F, Li C, Maechler M, Rossini AJ, Sawitzki G, Smith C, Smyth G, Tierney L, Yang JY, Zhang J: Bioconductor: open software development for computational biology and bioinformatics. Genome Biol 2004, 5:R80.

14. Database of GEne SeT AnaLysis Toolkit from WebGestalt. [http://bioinfo, vanderbilt.edu/webgestalt/]

15. Database for Annotation, Visualization and Integrated Discovery (DAVID). [http://david.abcc.ncifcrf.gov/].

16. Biological data analysis software from Ingenuity. [http://www.ingenuity. com].

17. Su Al, Wiltshire T, Batalov S, Lapp H, Ching KA, Block D, Zhang J, Soden R, Hayakawa M, Kreiman G, Cooke MP, Walker JR, Hogenesch JB: A gene atlas of the mouse and human protein-encoding transcriptomes. Proc Natl Acad Sci USA 2004, 101:6062-6067.

18. Lyck R, Ruderisch N, Moll AG, Steiner O, Cohen CD, Engelhardt B, Makrides V, Verrey F: Culture-induced changes in blood-brain barrier transcriptome: implications for amino-acid transporters in vivo. $J$ Cereb Blood Flow Metab 2009, 29:1491-1502.

19. HUGO Gene Nomenclature Committee database. [http://www. genenames.org/].

20. Database for Functional Analysis of Transcriptional Networks (FunNet). [http://www.funnet.info].

21. Palha JA, Nissanov J, Fernandes R, Sousa JC, Bertrand L, Dratman MB, Morreale de Escobar G, Gottesman M, Saraiva MJ: Thyroid hormone distribution in the mouse brain: the role of transthyretin. Neuroscience 2002, 113:837-847.

22. Sousa JC, de Escobar GM, Oliveira P, Saraiva MJ, Palha JA: Transthyretin is not necessary for thyroid hormone metabolism in conditions of increased hormone demand. J Endocrinol 2005, 187:257-266.

23. Obregon MJ, Mallol J, Escobar del Rey F, Morreale de Escobar G: Presence of L-thyroxine and 3,5,3'-triiodo-L-thyronine in tissues from thyroidectomized rats. Endocrinology 1981, 109:908-913.

24. Dohan O, De la Vieja A, Paroder V, Riedel C, Artani M, Reed M, Ginter CS, Carrasco N: The sodium/iodide Symporter (NIS): characterization, regulation, and medical significance. Endocr Rev 2003, 24:48-77.

25. Waksman G, Hamel E, Fournie-Zaluski MC, Roques BP: Autoradiographic comparison of the distribution of the neutral endopeptidase "enkephalinase" and of mu and delta opioid receptors in rat brain. Proc Natl Acad Sci USA 1986, 83:1523-1527.

26. Mansour A, Khachaturian H, Lewis ME, Akil H, Watson SJ: Autoradiographic differentiation of $\mathrm{mu}$, delta, and kappa opioid receptors in the rat forebrain and midbrain. J Neurosci 1987, 7:2445-2464.

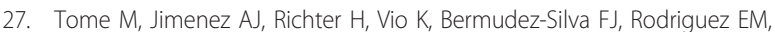
Perez-Figares JM: The subcommissural organ expresses D2, D3, D4, and D5 dopamine receptors. Cell Tissue Res 2004, 317:65-77.

28. Hu H: Chemorepulsion of neuronal migration by Slit2 in the developing mammalian forebrain. Neuron 1999, 23:703-711.

29. Nguyen-Ba-Charvet KT, Picard-Riera N, Tessier-Lavigne M, Baron-Van Evercooren A, Sotelo C, Chedotal A: Multiple roles for slits in the control of cell migration in the rostral migratory stream. J Neurosci 2004, 24:1497-1506.

30. Chodobski A, Szmydynger-Chodobska J: Choroid plexus: target for polypeptides and site of their synthesis. Microsc Res Tech 2001, 52:65-82.

31. Emerich DF, Skinner SJ, Borlongan CV, Vasconcellos AV, Thanos CG: The choroid plexus in the rise, fall and repair of the brain. Bioessays 2005, 27:262-274.
32. Bondy C, Werner H, Roberts CT Jr, LeRoith D: Cellular pattern of type-I insulin-like growth factor receptor gene expression during maturation of the rat brain: comparison with insulin-like growth factors I and II. Neuroscience 1992, 46:909-923.

33. Koo H, Choi BH: Expression of glial cell line-derived neurotrophic factor (GDNF) in the developing human fetal brain. Int I Dev Neurosci 2001, 19:549-558.

34. Diaz-Ruiz C, Perez-Tomas R, Domingo J, Ferrer I: Immunohistochemical localization of transforming growth factor-alpha in choroid plexus of the rat and chicken. Neurosci Lett 1993, 164:44-46.

35. Gonzalez AM, Berry M, Maher PA, Logan A, Baird A: A comprehensive analysis of the distribution of FGF-2 and FGFR1 in the rat brain. Brain Res 1995, 701:201-226.

36. Bagnard D, Vaillant $C$, Khuth ST, Dufay N, Lohrum M, Puschel AW, Belin MF, Bolz J, Thomasset N: Semaphorin 3 A-vascular endothelial growth factor165 balance mediates migration and apoptosis of neural progenitor cells by the recruitment of shared receptor. J Neurosci 2001, 21:3332-3341.

37. Vaskovsky A, Lupowitz Z, Erlich S, Pinkas-Kramarski R: ErbB-4 activation promotes neurite outgrowth in PC12 cells. J Neurochem 2000, 74:979-987.

38. Talmage DA: Mechanisms of neuregulin action. Novartis Found Symp 2008 , 289:74-84, discussion 84-93.

39. Zapala MA, Hovatta I, Ellison JA, Wodicka L, Del Rio JA, Tennant R, Tynan W, Broide RS, Helton R, Stoveken BS, Winrow C, Lockhart DJ, Reilly JF, Young WG, Bloom FE, Lockhart DJ, Barlow C: Adult mouse brain gene expression patterns bear an embryologic imprint. Proc Natl Acad Sci USA 2005, 102:10357-10362.

40. Zlokovic BV: The blood-brain barrier in health and chronic neurodegenerative disorders. Neuron 2008, 57:178-201.

doi:10.1186/2045-8118-8-10

Cite this article as: Marques et al:: Transcriptome signature of the adult mouse choroid plexus. Fluids and Barriers of the CNS 2011 8:10.

\section{Submit your next manuscript to BioMed Central and take full advantage of:}

- Convenient online submission

- Thorough peer review

- No space constraints or color figure charges

- Immediate publication on acceptance

- Inclusion in PubMed, CAS, Scopus and Google Scholar

- Research which is freely available for redistribution 